\title{
Piomiositis Informe de 132 pacientes
}

\author{
Campbell S.', Iglesias A. ${ }^{2}$
}

\section{Resumen}

Fundamento. La infección bacteriana del músculo estriado, acompañado por la formación de abscesos, es una situación excepcional. Evaluamos las características clinicas de la piomiositis a fin de favorecer un diagnóstico y tratamiento más tempranas.

\begin{abstract}
Métodos. Se estudiaron 132 pacientes afectados de piomiositis, aiendidosen los últimos 9 años. Los criterios de inclusión fueron: 1) clínica compatible; 2) demostración de un absceso mediante punción o drenaje; 3) evidencia de dolor muscular local acompañado o no de tumefacción o masa y cuya respuesta clinica favorable se deba única y exclusivamente a la oxacilina, así no se haya practicado punción o drenaje.
\end{abstract}

Resultados. La edad media de los pacientes estudiados fue de 21,5 años (rango: 15 meses a 68 años). Se produjo la enfermedad en 88 varones $(66,6 \%)$. El tiempo de evolución clínica previo a la consulta osciló entre 2 y 94 días, para un promedio de 11,6. La fiebre fue el síntoma predominante $(98,4 \%)$ mientras que la presencia de masa 0 tumefacción fue el signo más notorio $(97,7 \%)$. El dolor se encontró en el $(96,9 \%)$ de los casos. El antecedente traumático aparece en 25 pacientes $(18,9 \%)$ y el de piodermitis en $64(48,4 \%)$. Los músculos más afectados fueron los de los miembros inferiores (47\%) y, en su orden: muslos, pantorrillas y glúteos. Aunque el absceso agudo fue la forma de presentación clínica más frecuente $(82,5 \%)$, se encontró que no todas las lesiones evolucionan aabscesos y se identificó una forma más de presentación en tres pacientes $(2,3 \%)$ : el dolor muscular localizado, sin masa ni tumefacción, acompañado de fiebre. La masa dura sin absceso $(10,7 \%)$ y la forma blanda con supuración $(4,5 \%)$ fueron las otras maneras de manifestarse la enfermedad. Ctaphylococcus aureus coagulasa (+) fue el germen más frecuentemente aislado en e! sitio de las lesiones $(96,7 \%)$, seguido por Staphylococcus epidermidis $(2,2 \%)$ y Pseudomonas aeruginosa $(1,1 \%)$. Las complicaciones observadas fueron: la neumonía estafilocócica $(1,5 \%)$ y la artritis séptica $(0,7 \%)$. Todos los pacientes curaron con el tratamiento médico.

Conclusiones. 1) La piomiositis es frecuente en nuestro medio; 2) es la serie más grande publicada en Colombia y en América; 3) se encontraron tres casos de dolor muscular local y fiebre como una forma más de presentación clinica; 4) se identificó un caso de Pseudomonas aeruginosa como agente colonizador del músculo; 5) el antecedente de trauma se encontró en el $18 \%$ de los casos; 6 ) la forma de presentarse la enfermedad no corresponde siempre con la fase evolutiva descrita; $y, 7$ ) independiente del germen causal, la enfermedad sigue un curso clínico semejante.

\footnotetext{
Médico internista, Servicio de Medicina Interna, Hosptal María Inmaculada, Forencia, Caquetá.

2 Médico reurnatólogo. Universidad Nacional; Instituto Nacional de Salud. Santafé de Bogotá, D.C.
} 


\section{Summary}

Background. Bacterial infection of striated muscle accompanied by abscess formation is an unusual situation. We evaluated the clinical features of pyomyositis in order to make an earlier diagnosis and treatment possible.

Method. A sample of one hundred and thirty-two patients suffering from pyomyositis seen over the last 9 years was studied. Inclusion criteria were: 1) compatible clinical manifestations; 2) demonstration of an abscess through puncture or drainage; 3 ) evidence of local muscle pain, with or without swelling or mass, and the favourable clinical response due exclusively to oxacillin, regardless of puncture or drainage having been made.

Results. Mean age of the patients in the study was 21.5 years (range: 15 months to 68 years). The condition was present in 88 males $(66.6 \%)$. The onset of clinical manifestations prior to consultation was between 2 and 94 days, with an average of 11.6. The most frequent symptom was fever $(98.4 \%)$ while the most noticeable sign was the presence of a mass or swelling $(97.7 \%) .96 .9 \%$ of the subjects reported pain. Previous trauma appeared in $25(18.9 \%)$ patients and pyodermitis in another 64 $(48.4 \%)$. The most affected muscles were those of the lower limbs $(47 \%)$ i.e. thighs, calves and buttocks. Although the presence of acute abscess was the most common clinical ocurrence $(82.5 \%)$, it was found that not all lesions evolved into abscesses, and a new presentation was identified in three patients $(2.3 \%)$ - localized muscle ache without mass or tumescence with fever. The hard mass without abscess $(10.7 \%)$ and soft mass with suppuration (4.5\%) were the other clinical occurrences. Staphylococcus aureus coagulase $(+)$ was the most frequent germ isolated at the site of the lesions (96.7\%), followed by Staphylococcus epidermidis (2.2\%) and Pseudononas aeruginosa $(1.1 \%)$. Complications were: staphylococcal pneumonia $(1.7 \%)$ and septic arthritis $(0.7 \%)$. All patients healed with appropriate medical treatment.

Conclusions. 1) Pyomyositis is a frequent disease in our country. 2) Up to date, this is the greatest number of cases published both in Colombia and in America. 3) Three cases of local pain and fever as a further way of clinical presentation were found. 4) A case of Pseudomonas aeruginosa as a muscle colonizer agent was evidenced. 5) Prior trauma was found in $18 \%$ of the cases. 6) The presentation of the disease does not always correspond to the evolutive stage herein described. 7) The disease follows a similar clinical course non-dependent from the causative microorganism.

La piomiositis puede definirse como la formación de abscesos intra o intermusculares, ocasionados la mayoría de las veces por la colonización del músculo esquelético porStaphylococcus aureus coagulasa positiva, hecho que puede desencadenarse de manera espontánea, por trauma o por picaduras de insectos. Se debe excluir la miositis causada por heridas penetrantes o por extensión directa de infecciones vecinas (1).

Las primeras referencias que se tienen de estos abscesos datan de 1852 en descripciones hechas por Virchow en Europa, según el cirujano japonés Miyake (2). En 1858, Gellé describió dos casos en Francia (3) y Scriba en 1885 realizó la primera información de una serie de pacientes vistos en un corto período de tiempo y pensó que su origen se relacionaba en zonas tropicales (4). La serie más grande publicada en el mundo es la de Horn y Master en 1968 quienes describieron 800 casos en Uganda (5). En América, los primeros casos se publicaron en 1866 por De Salvia en el Brasil (6) y la serie más extensa es la de Kerrigan y Nelson en el Ecuador oriental, conformada por 97 pacientes reunidos durante 10 años (7). 
En Colombia, en 1980, Iglesias y Pineda, Barón y Rodríguez describieron los primeros nueve casos observados durante seis años $(8,22)$. La información nacional más reciente estudió 75 pacientes, el grupo más grande informado en nuestro medio (9).

La piomiositis tiene una distribución mundial, pero, predomina en zonas tropicales con climas cálidos y húmedos y con altitudes inferiores a los $1500 \mathrm{msnm}$. Sin embargo, como se han descrito casos en sitios como Londres (10), Boston (11), Sydney (12), Chicago (13) y Santafé de Bogotá $(8,14)$ se ha llegado a pensar que no es una enfermedad de estos climas ni parece que ellos sean factores determinantes en el inicio de la entidad $(8,15)$.

En este informe se expone nuestra experiencia con la piomiositis y se pretende contribuir al conocimiento de esta patología que tiene un buen pronóstico cuando su identificación es temprana y el tratamiento oportuno y adecuado, a pesar de la potencial agresividad de los gérmenes encontrados.

\section{Pacientes y métodos}

Serie clínica descriptiva llevada a cabo en el Hospital María Inmaculada de Florencia, Caquetá entre enero de 1986 y diciembre de 1993. Se obtuvieron 158 historias de la sección de estadística con diagnóstico de pıomiositis. Se seleccionaron 132 con base en uno o más de los siguientes criterios:

1. Historias que se ajustaran con la definición anotada.

2. Historias que, además de la presencia de material purulento obtenido por punción o drenaje de masas o tumefacciones musculares, informaran sobre la existencia de microorganismos en la tinción de Gram o en el cultivo.

3. Historias con aspirado por punción positiva para material purulento de masas o tumefacciones musculares, pero, con Gram o cultivo sin demostrar gérmenes.

4. Historias con aspirado por punción y/o drenaje positivo para material purulento de masas o tumefacciones musculares, así el Gram o el cultivo no aparecieran informados.

5. Historias que, además del cuadro febril, informaran sobre la existencia de dolor muscular localizado que podía o no estar acompañado de tumefacción o masa, pero sin evidencia de otra enfermedad de base o concomitante y cuya respuesta favorable se deba única y exclusivamente a la oxacilina así no se haya practicado punción o drenaje.

Las historias seleccionadas se estudiaron en su totalidad.

\section{Resultados}

De los 158 pacientes que inicialmente se incluyeron en el estudio, 26 fueron excluidos por diversas razones: algunos no correspondían con la definición dada (eran infecciones del tejido celular subcutáneo) y, en otros, los datos no eran precisos o eran incompletos y no permitían tener una definición clara del problema.

La mayoría de las historias pertenecían a pacientes hospitalizados en las salas generales $(87,8 \%)$, con nivel socioeconómico bajo. La enfermedad predominó en personas jóvenes con una edad que osciló entre 15 meses a 68 años, con promedio de 21,5 años (figura 1). El sexo masculino fue el más comprometido, relación 2:1. En el momento de la consulta la mayoría de los pacientes $(78 \%)$ se encontraban en la segunda semana de haberse iniciado los primeros signos o síntomas

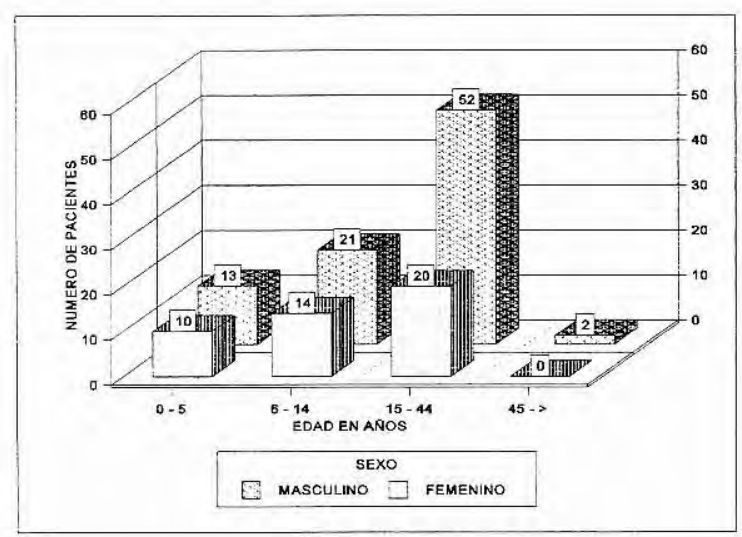

Figura 1. Distribución de los pacientes con piomiositis según edad y sexo. 
(promedio de 11,6 días con rango de 2 a 94) y el $61,3 \%$ habían recibido antibióticos, siendo las penicilinas las más utilizadas (68\%). Un $46,3 \%$ consultaron en los meses de febrero, marzo y abril. El $56,1 \%$ de los pacientes provenían de diferentes áreas rurales del departamento y el $35,6 \%$ de diversos cascos urbanos sin poder definir una zona específica. El $8,3 \%$ eran de zonas rurales del Putumayo.

Los días de hospitalización tuvieron un promedio de $10,8 \%$ con rango de 5 a 25 días. En 96 pacientes $(72,7 \%)$ el diagnóstico se hizo desde el ingreso.

La fiebre fue el síntoma predominante $(98,4 \%)$ mientras que la presencia de masa o tumefacción fue el signo más notorio $(97,7 \%)$. La temperatura osciló entre 38 y $41^{\circ} \mathrm{C}\left( \pm \mathrm{DE}=39 \pm 0,8^{\circ} \mathrm{C}\right)$. El antecedente de trauma sólo aparece en 25 pacientes $(18,9 \%)$ mientras que el de piodermitis está en $64(48,4 \%)$ (tabla 1). El hemicuerpo derecho tuvo un leve predominio sobre el izquierdo (figura 2) y fue más evidente el compromiso en los grupos musculares de los miembros inferiores (figura 3) y en su orden se encontraron más comprometidos los músculos de los muslos, pantorrillas y glúteos. En las extremidades superiores fue más evidente el compromiso del deltoides, bíceps braquial, braquial anterior y tríceps; la musculatura paravertebral, los músculos pectorales y los rectos abdominales fueron los más frecuentes del tronco.

Tabla 1. Distribución de los pacientes según hallazgos clínicos.

\begin{tabular}{|c|c|c|}
\hline Hallazgo & Pacientes & Porcentaje \\
\hline Fiebre & 130 & 98,48 \\
\hline Masa & 129 & 97,73 \\
\hline Dolor & 128 & 96,97 \\
\hline Signos inflamatorios* & 104 & 78,79 \\
\hline Impotencia funcional & 74 & 56,06 \\
\hline Piodermitis ** & 64 & 48,48 \\
\hline Dos o más abscesos & 42 & 31,82 \\
\hline Trauma ${ }^{\star \star}$ & 25 & 18,94 \\
\hline Absceso del psoas & 6 & 4,55 \\
\hline
\end{tabular}

* Calor, edema y eritema local

** Antecedentes

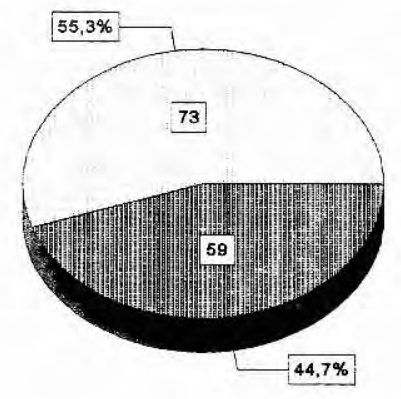

Numero de pacientes: 132 DERECHO 闻 IZQUIERDO

Figura 2. Distribución de los pacientes según el hemicuerpo comprometido.
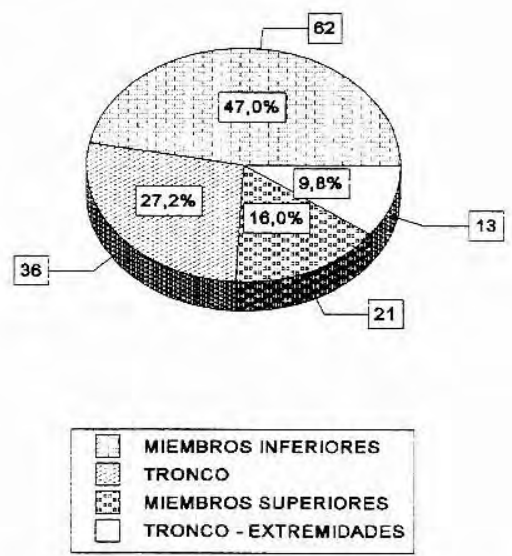

Figura 3. Ubicación corporal del compromiso muscular.

El absceso agudo fue la forma de presentación clínica más frecuente $(82,5 \%)$, manifestado por masa muscular con dolor pulsátil y fiebre acompañado de hipertermia y eritema local. En segundo lugar, se encontró la presencia de masa dura, leñosa $(10,7 \%)$, que en doce casos se acompañó de dolor de intensidad variable y fiebre; en un paciente, el endurecimiento muscular fue el único hallazgo mientras que el otro tuvo fiebre pero $\sin$ dolor. En seis pacientes $(4,5 \%$, ) la masa se acompañó de gran fluctuación y renitencia, cinco tuvieron fiebre y, uno de éstos, la tumefacción era indolora; en el otro fue completamente asintomática. 
En menor proporción (2,3\%) se hallaron tres pacientes con mialgias localizadas y fiebre, pero, sin signos de fluctuación o endurecimiento (tabla 2).

La velocidad de sedimentación globular elevada y la leucocitosis fueron los hallazgos más notorios entre los paraclínicos con un $97,7 \%$ y $94,6 \%$ respectivamente; mientras que la eosinofilia se registró en un $28,7 \%$ y la anemia en un $24,2 \%$. Las enzimas que se determinaron mostraron un porcentaje menor de alteración (tabla 3). No se practicaron hemocultivos.

Unicamente en ocho pacientes $(6 \%)$ se practicó biopsia muscular, dos de las cuales pertenecían a pacientes con masa dura y otra a un paciente con mialgia localizada; las cinco restantes co-

Tabla 2. Formas de presentación clínica.

\begin{tabular}{lrc}
\hline \multicolumn{1}{c}{ Formas } & Pacientes & Porcentaje \\
\hline Absceso agudo & 109 & 82,57 \\
Masa dura & 14 & 10,61 \\
Masa blanda & 6 & 4,55 \\
Dolor localizado sin masa & 3 & 2,27 \\
\hline \multirow{2}{*}{ Total } & & \\
& 132 & 100,00
\end{tabular}

Tabla 3. Hallazgos paraclínicos.

\begin{tabular}{lcc}
\hline \multicolumn{1}{c}{ Parámetro } & Pacientes & Porcentaje \\
\hline VSG elevada* $^{*}$ & 129 & 97,73 \\
Leucocitosis $^{\star \star}$ & 125 & 94,70 \\
Eosinofilia $^{\dagger}$ & 38 & 28,79 \\
Anemia $^{\ddagger}$ & 32 & 24,24 \\
CK total elevada $^{5}$ & 16 & 12,12 \\
AST elevada $^{*}$ & 11 & 8,33 \\
DHL elevada $^{\natural}$ & 8 & 6,06 \\
\hline
\end{tabular}

\footnotetext{
* VSG $>20 \mathrm{~mm} / \mathrm{h}$ (Wintrobe)

** Leucocitos $>10 \times 10^{\circ} / \mathrm{L}$

+ Eosinófilos $>0,5 \times 10 \%$

$\neq \mathrm{Hb}<6 \mathrm{mmol} / \mathrm{L}$

\& CK normal hasta $1,18 \mathrm{mmol} / \mathrm{s} / \mathrm{L}$

AST normal hasta $300 \mathrm{umol} / \mathrm{s} / \mathrm{L}$

s DHL normal hasta $1,7 \mathrm{mmol} / \mathrm{s} / \mathrm{L}$
}

rrespondieron a abscesos agudos. La histopatología mostró severo compromiso inflamatorio con focos hemorrágicos, separación de las fibras musculares, algunas de ellas con pérdida de la estriación, otras son vacuoladas y en proceso de lisis. El infiltrado predominante es de histiocitos, PMN y eosinófilos. En tres se informaron abscesos confluentes con acúmulos de PMN y cocos Gram positivos; existía, además, trombosis venular y capilares engrosados.

La ecografía como ayuda diagnóstica o para dirigir la punción se practicó en 22 pacientes $(16,6 \%)$ y en 16 casos mostró zona anecoica con edema perilesional; en las restantes no hubo alteración.

En 98 pacientes $(57,3 \%)$ se obtuvo material purulento, cremoso, espeso e inoloro en lo más profundo del músculo (figura 4). La tinción de Gram se practicó en todos, identificándose gérmenes en 96, mientras que el cultivo mostró crecimiento bacteriano en 90 casos, siendo el Staphylococcus aureus coagulasa positivo el microorganismo más frecuente (tabla 4). En ocho muestras no creció ninguna bacteria a pesar de que el Gram mostró cocos Gram (+) en seis. En los otros dos casos ninguno de los dos métodos identificó gérmenes.

En nueve casos el aspirado por punción no obtuvo ningún material y en 25 no se practicó punción ni drenaje quirúrgico obteniéndose buena respuesta con el antibiótico empleado (tabla 5).

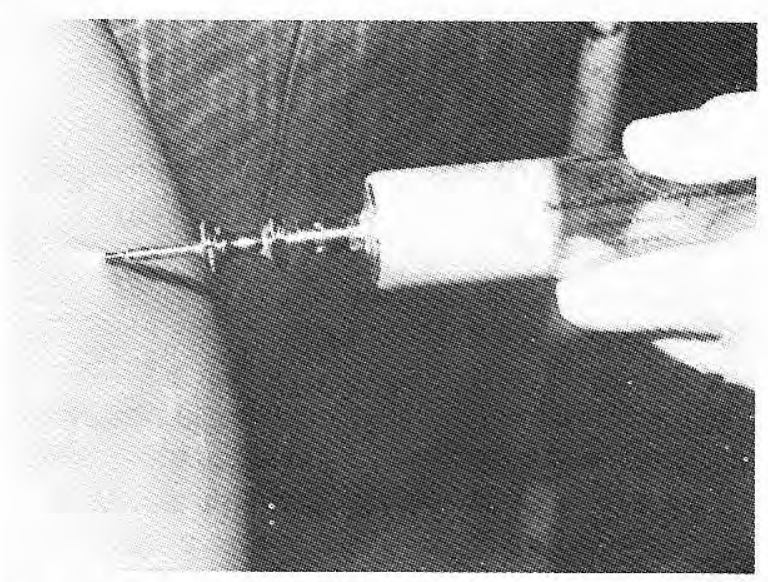

Figura 4. Aspecto del pus obtenido por puncion. 
Tabla 4. Distribución de los pacientes según el método diagnóstico empleado para la identificación bacteriana.

\begin{tabular}{lcc} 
TINCION DE GRAM & PACIENTES & PORCENTAJE \\
\hline Cocos grampositivos & 95 & 96,94 \\
Sin gérmenes & 2 & 2,04 \\
Bacilos gramnegativos & 1 & 1,02 \\
\hline Total & 98 & 100,00 \\
\multicolumn{1}{c}{ CULTIVO $* *$} & PACIENTES & PORCENTAJE \\
\hline
\end{tabular}

\begin{tabular}{lcc}
$\begin{array}{l}\text { S. aureus } \\
\text { coagulasa positiva }\end{array}$ & 87 & 88,78 \\
S. epidermidis & 2 & 2,04 \\
$P$. aeruginosa & 1 & 1,02 \\
Negativos & 8 & 8,16 \\
\hline & 98 & \\
Total & \\
\hline * El cultivo también fue negativo & \\
** Para gérmenes aerobios comunes
\end{tabular}

Tabla 5. Motivo por el cual no se aislaron bacterias en 42 pacientes.

FACTOR CAUSAL PACIENTES PORCENTAJE

\begin{tabular}{lcc}
$\begin{array}{l}\text { Sin procedimiento } \\
\text { quirúrgico* }\end{array}$ & 25 & 59,52 \\
$\begin{array}{l}\text { Aspirado por } \\
\text { punción negativo }\end{array}$ & 9 & 21,43 \\
Cultivo negativo & 8 & 19,05 \\
\hline Total & 42 & 100,00 \\
\hline
\end{tabular}

* Punción evacuadora o drenaje abierto

** Para gérmenes aerobios comunes.

La complicación más frecuente fue la neumonía estafilocócica encontrada en dos pacientes (1,5\%). Uno de ellos inició el cuadro pulmonar dos días antes del ingreso al hospital y la enfermedad había evolucionado durante 42 días. El otro paciente presentó la complicación cuatro días des- pués del ingreso. En este paciente y en el que presentó la artritis séptica en la rodilla izquierda $(0,7 \%)$, la enfermedad había evolucionado durante 23 y 29 días, respectivamente, como una masa dura, pétrea y habían recibido inicialmente penicilina.

El tratamiento se hizo con oxacilina parenteral en todos los pacientes durante una a dos semanas. En los adultos, la dosis fluctuó entre 6 y $12 \mathrm{~g} / \mathrm{d}$ ía y en los niños se dosificó a razón de $150 \mathrm{mg} / \mathrm{kg} /$ día. Un paciente ameritó gentamicina por falta de respuesta al tratamiento; el cultivo informó Pseudomonas aeruginosa. Todos respondieron favorablemente al manejo médico empleado y no se registraron recaídas. El cumplimiento del tratamiento ambulatorio no se pudo documentar con precisión.

No se documentaron reingresos por la misma patología ni hubo antecedentes de hechos similares. No se encontró evidencia de otra enfermedad de base (neoplasia, diabetes mellitus) o inmunosupresión por drogas. Todos aparentaban buen estado previo. Es importante anotar que cinco pacientes presentaron simultáneamente paludismo (cuatro por Plasmodiun vivax y uno por Plasmodiun falciparum).

\section{Discusión}

La piomiositis ha sido informada infrecuentemente en Colombia. De las siete publicaciones que existen en nuestro medio (tabla 6), cuatro (8, $9,14,16$ ) incluyeron pacientes procedentes de diferentes áreas del Caquetá. Realmente sorprende la alta presencia de la enfermedad en esta zona del país y hay que reconocer que sólo se buscaron aquellas historias cuyo diagnóstico final fue de piomiositis; sin embargo, posteriormente pudimos darnos cuenta que algunas historias clasificadas como simples abscesos, correspondían a la entidad requerida. Es probable que en otras zonas también sea frecuente, pero no hay información nacional.

Es importante recordar que el músculo esquelético presenta una resistencia inherente a la invasión bacteriana. La presencia de abscesos musculares es una situación excepcional aún en casos de sepsis severa de diferentes etiologías. 
Estudios como los de Duma (53) y Churchill (54) no pudieron demostrar ningún absceso en casos de bacteremia por estreptococos. Se ha considerado al trauma como factor predisponente para la colonización bacteriana. Esta apreciación parte de los estudios de Miyake en 1904 (2) y de Halsted en 1924 (52) quienes demostraron que para que se produzca un absceso muscular mediante la administración endovenosa de una dosis subletal de Staphylococcus aureus, se necesita lesionar previamente el músculo por pinzamiento mecánico o estimulación eléctrica; sin embargo, el antecedente traumático no es constante en todos los casos.

En cuanto a la edad y sexo no hay diferencias significativas con otras publicaciones $(8,11,15,17,18$, 21). La mayoría de los pacientes consultaron en la segunda semana de la enfermedad; sin embargo, un paciente prolongó su cuadro febril por 94 días acompañado sólo de dolor leve sobre el deltoides derecho. Esto hace pensar que la enfermedad puede presentarse como un síndrome febril prolongado. Curiosamente este paciente de manera empírica recibió varios tratamientos antipalúdicos por prescripción y automedicación, lo mismo que cloramfenicol por supuesta fiebre tifoidea. Los síntomas vagos y prolongados retardan el diagnóstico; pueden persistir hasta un año (57).

También sorprende el porcentaje de pacientes que reciben antibióticos sin una clara indicación $(61,3 \%)$ sobresaliendo la ampicilina entre ellos, seguidos por el trimetropim-sulfametoxazol y la norfloxacina. No hay una explicación adecuada para la mayor incidencia de la enfermedad en los meses de febrero, marzo y abril. Esta época se relaciona con el inicio del invierno y los casos de paludismo aumentan en esta región. Un informe ha propuesto a los mosquitos como agentes transmisores de virus, que predispondrían al músculo para ser colonizado porel Staphylococcus aureus (19), pero, llama la atención que en ciertos brotes epidémicos informados, algunas razas no sean afectadas $(20,21)$ y un estudio nacional (22) y otros $(5,17,48,55)$ no lograron demostrar partículas virales. Hay una asociación descrita con el virus de Epstein-Barr (58).

Es llamativo en este estudio la baja frecuencia del antecedente traumático; sólo se registró en un $18,9 \%$ de los casos. Este antecedente no es uniforme en los diferentes estudios, las variaciones van desde el $20 \%$ (50) hasta el $83 \%(8,23$, 24). Es probable que el traumatismo no tenga un papel importante en la presentación de nuestros casos aunque siga considerándose como posible factor etiológico (25). La posibilidad de un traumatismo banal que pase desapercibido por el paciente no se puede descartar, pero este tipo de trauma se sucede a diario en todos los sexos, razas, edades y climas y, sin embargo, los abscesos musculares no se ven con esa frecuencia.

Como es conocido, son las defensas del huésped las que limitan y suprimen los fenómenos infecciosos. Estas defensas comprenden la piel, la respuesta inflamatoria del sistema humoral, la inmunidad debida a células y el complemento. La piel, además de constituirse en una excelente barrera mecánica por sus capas fundamentales, cumple funciones inmunológicas específicas por parte de varias de sus células, entre ellas los

Tabla 6. Cronología de las publicaciones de piomiositis en Colombia.

\begin{tabular}{cllr}
\hline AÑo & \multicolumn{1}{c}{ AUTOR } & \multicolumn{1}{c}{ TEMA } & REFERENCIAS \\
\hline 1980 & Iglesias y Pineda & Descripción de 6 casos & 8 \\
1980 & Barón y Rodríguez & Descripción de 3 casos & 22 \\
1987 & Rodríguez, Saravia y col. & Recopilación de 11 casos & 14 \\
1988 & Ramírez y Ruiz & Recopilación de 8 casos & 33 \\
1988 & Varela, Marulanda y col. & Descripción de 1 caso & 16 \\
1990 & Ramírez y Ruiz & Complemento de 12 casos & 18 \\
1991 & Campbell & Informe 75 casos & 9 \\
\hline
\end{tabular}


queratinocitos que tienen similitud morfológica y funcional con las células epiteliales del timo y participan en la maduración de los linfocitos $T$. Las células de Langerhans que presentan en su membrana antígenos HLA-DR y receptores Fc para el complemento entre otros y las células de Granstein, subpoblación de CD, que en contacto con los linfocitos $T$ inducen la formación de $L T$ supresores. En el caso eventual que este factor protector se perdiera por un trauma, para que el germen alcance y colonize el músculo se necesita que el sistema defensivo falle y, en especial, el sistema fagocitario (PMN) y el adaptativo (macrófagos-linfocitos T yB) y que, además, exista bacteremia o miofilia especial por el germen. Sin embargo, los pacientes con esta enfermedad generalmente tienen un buen estado de salud previo como se pudo determinar en este informe y por otros estudios (23) y aunque ya hace tiempo se informó sobre las variaciones periódicas de las funciones bactericidas de los neutrófilos (26) actualmente no se han demostrado alteraciones en ellos (27) ni inmunodeficiencias (8), aunque cabe anotar que un grupo de pacientes nigerianos tenían un nivel significativamente bajo de IgG $(28,29)$ y el trabajo de idoko $(27)$ mostró una ineficiencia en los linfocitos $T$. Creemos que estos hallazgos y su relación directa con la piomiositis no son concluyentes porque como se anotó anteriormente, otros estudios no tuvieron hallazgos similares.

La multiplicidad de los abscesos, que en nuestra serie fue de $31,8 \%$, es un hecho que estaría a favor de una bacteremia previa pero en contra de un trauma local único. Encontramos que doce pacientes $(9 \%)$ tuvieron más de dos abscesos simultáneos en la misma fase evolutiva y sin antecedentes de trauma. Sólo en el $5 \%$ de los pacientes que presentan la enfermedad se ha determinado bacteremia en los hemocultivos (34); sin embargo, la serie española más amplia informa un $79 \%$ en los casos que ellos consideraron primarios (50).

La miofilia del germen es el otro factor involucrado en la patogénesis de la enfermedad, pero, no se han encontrado fagotipos específicos (18). Se ha propuesto que a mayor actividad de lipasa por parte del Staphylococcus aureus mayor com- promiso profundo (30-32). Es posible que el trauma, aunque puede ser un factor contribuyente en algunos casos, por sí solo no sea condición sufi-ciente para explicar el desarrollo de la enfermedad. En cambio, las alteraciones del microambiente local de la piel en casos de lesiones piodermíticas permitirían una puerta de entrada más factible que la ruptura inaparente de la epidermis por traumas leves, locales, aislados y no repetitivos. En nuestra serie, el antecedente existió en un $48,4 \%$, más alto que el trauma. En un estudio nacional se encontró en un 8,3\% (18), pero, otros lo han encontrado hasta en un $55 \%$ (25). Es importante anotar que en nuestros casos existía el antecedente de estas lesiones y la ubicación del absceso muscular no coincidía con el sitio anatómico de la cicatriz piodermítica.

La patogenia de la infección del tejido muscular estriado sigue siendo desconocida y aunque se han involucrados factores predisponentes, ninguno de ellos lo es en forma convincente, incluyendo a la microisquemia muscular (56).

El Staphylococcus aureus coagulasa positiva es la bacteria que con mayor frecuencia se aisla; sin embargo, la gama de patógenos aumenta cada día (36-43). Las características clínicas descritas no demuestran diferencias entre ellos ni con el Staphylococcus aureus, hecho que corroboramos nosotros. Con la pandemia del síndrome de inmunodeficiencia adquirida (SIDA) se ha descrito un mayor número de casos estableciéndose una predisposición para padecer de piomiositis. En los casos descritos se ha observado un curso subagudo y en la mayoría de los pacientes los hemocultivos han sido negativos (51).

En cuanto a la ubicación corporal, el lado derecho dominante del cuerpo se ha implicado con una frecuencia dos a tres veces superior a la del izquierdo $(13,34)$; probablemente este hecho tampoco sea importante entre nosotros porque esta diferencia no se observó (figura 2). El compromiso más notorio en los grupos musculares de los miembros inferiores es posible que se deba a una mera consecuencia de su tamaño.

Se ha considerado que la evolución usual de la enfermedad es de dos a cuatro semanas aunque 
puede prolongarse (23) y claramente se han definido tres fases $(17,23,24)$; la primera, que es la etapa invasiva, ocurre en la primera semana de la enfermedad y se caracteriza clínicamente por fiebre, dolor, limitación funcional y tumefacción o masa en el sitio afectado (figura 5); la segunda, período supurativo, corresponde a la segunda semana y por fenómenos de miocitólisis hay marcado edema, renitencia, dolor, fiebre y el tejido celular subcutáneo aparece eritematoso, en esta etapa el aspirado es positivo (figura 6). La tercera fase se ha caracterizado por la diseminación tanto por contigüidad como a distancia. En general, éste es el aspecto evolutivo de la enfermedad y en toda su expresión correspondería a un absceso muscular complicado; pero, hay que tener en cuenta que su evolución no siempre corresponde con la manera de presentarse clínicamente; por tanto, para su diagnóstico, que debe establecerse sobre una base exclusivamente clínica se debe tener en cuenta que la enfermedad tiene diferentes formas de presentación que aparentemente no siguen ese aspecto evolutivo.

Se reconocen tres formas de presentación (34): el absceso muscular agudo, el reemplazo muscular por una masa fluctuante sin dolor ni fiebre y el reemplazo por masa dura ligeramente dolorosa.

En esta serie identificamos una forma de presentación más: el dolor localizado a un grupo muscular sin masa y con fiebre. De este grupo, tres pacientes evolucionaron durante 24,37 y 94 días respectivamente sin poder definir en ningún momento tumefacción o masa por palpación y la ecografía no logró delimitar ninguna zona anecoica y sólo un paciente recibió tratamiento antipalúdico y clorafenicol. A este caso se le practicó biopsia muscular. A los otros, aunque no se les practicó, la repuesta a la oxacilina fue favorable y rápida. Igual resultado se obtuvo con otros dos pacientes con masa dura, leñosa y cuya evolución fue de 28 y 39 días respectivamente y sin uso previo de antibióticos. Esto nos ha permitido pensar que no se necesita la presencia de pus desde el punto de vista macroscópico para hacer el diagnóstico porque existen estas formas de presentación que no evolucionan a abscesos por alguna razón que en este informe no pudo precisar.

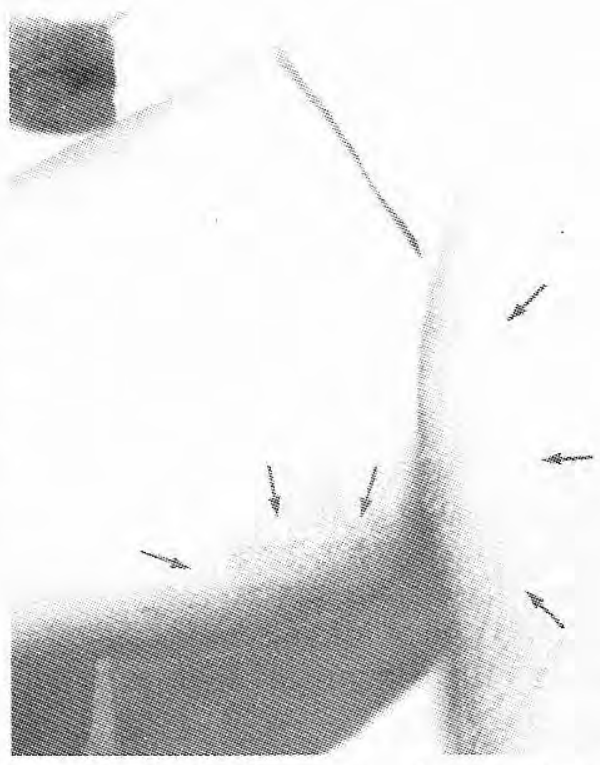

Figura 5. Piomiositis en primera fase. Se observa asimetría en la parte anterior del muslo izquierdo y en la posterior de derecho.

Con respecto a la fiebre, aunque fue el síntoma que se encontró en mayor porcentaje $(98,4 \%)$, dos pacientes no la presentaron; uno con masa dura en rectos abdominales y otro con gran zona fluctuante, renitente sobre el oblicuo externo derecho; de igual manera podemos decir que el dolor no siempre se presentó en los pacientes (cuatro no lo tuvieron). El estado general de los pacientes no se alteró en forma importante a pesar de tener abscesos de gran tamaño.

La leucocitosis con desviación a la izquierda y el aumento de la VSG fueron los hallazgos paraclínicos mas sobresalientes, lo cual concuerda con la experiencia nacional y con otros autores $(13,17,35,44)$. El material purulento que se obtuvo en 98 pacientes $(74,2 \%)$ sólo mostró bacterias en el cultivo en 90 casos. En seis casos la tinción de Gram mostró cocos grampositivos, pero, en el cultivo no creció ninguna bacteria; probablemente el uso previo de antibióticos influyó en la negativización de estas muestras como también en las otras dos que ninguno de los dos métodos identificó bacterias o quizás los medios diagnósticos empleados no eran los adecuados (tabla 5). En un paciente se identificó Pseudomonas aeruginosa; este germen como algunos estreptococos y los anaerobios pueden ocasionar in- 
fecciones necrotizantes severas del tejido celular subcutáneo y de las fascias, ocasionando mionecrosis agresivas, cuadro que no correspondió con el paciente en mención; por tanto, pensamos que es la primera vez que se identifica a Pseudomonas como agente colonizador del músculo. No hubo diferencias entre la presentación de la enfermedad y el agente bacteriano encontrado.

Es pertinente puntualizar que a los pacientes que no se les practicó drenaje quirúrgico repondieron favorablemente con el antibiótico utilizado; por tanto, creemos que algunos pacientes podrían no requerirlo sin demeritar nunca la importancia que tiene. Este hecho es más evidente cuando el diagnóstico se hace con prontitud. En esta revi-
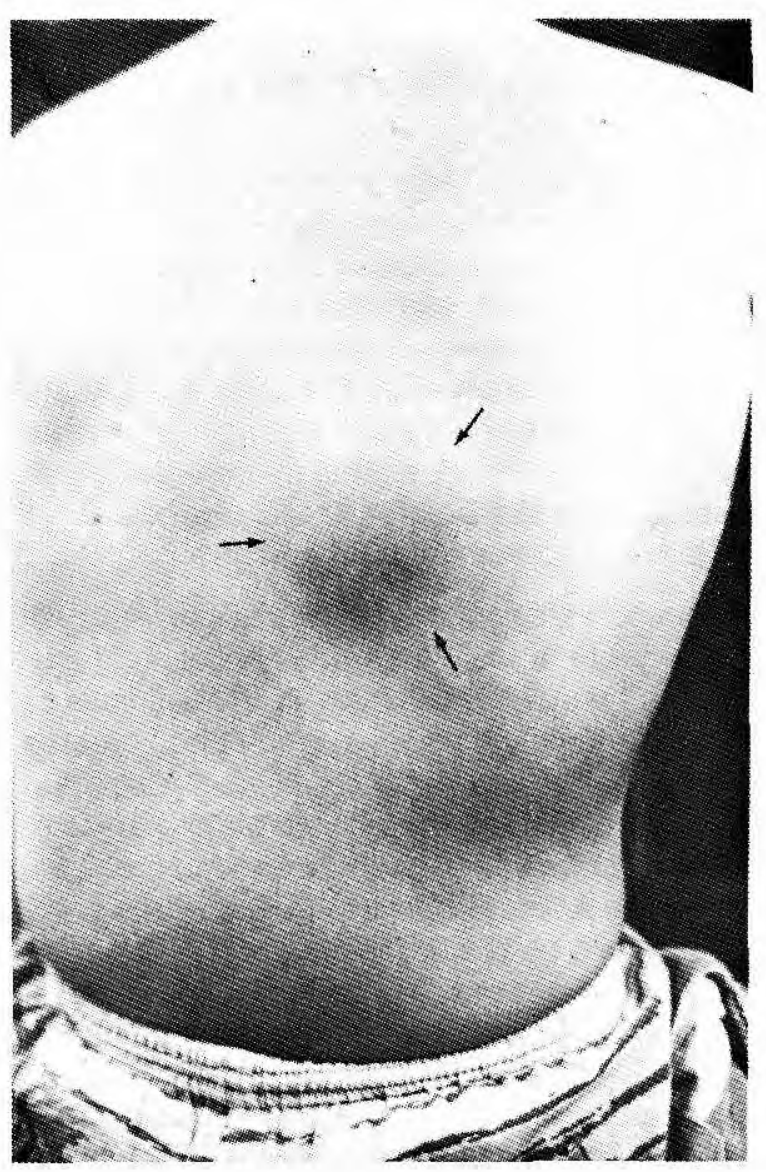

Figura 6. Piomiositis en segunda fase. Dos aspectos diferentes de la masa muscular en un mismo paciente: la que existe en el dorsal ancho tiene eritema local. sión el diagnóstico se hizo desde el ingreso en 96 pacientes $(72,7 \%)$ y denota la familiaridad que se tiene con esta patología. En cambio, hemos observado que puede convertirse en un verdadero reto diagnóstico para el médico no familiarizado aunque la forma de presentación clínica sea la de un absceso agudo. Es el verdadero conocimiento de la entidad lo que influyó para disminuir los días de hospitalización de nuestros pacientes $(\bar{X}=10.9)$ con respecto a otras publicaciones $(8,22,23)$. De igual manera, es el conocimiento de esta patología y su manejo oportuno lo que hará que las complicaciones serias y fatales en algunos casos no se presenten (4547).

De los resultados obtenidos podemos concluir:

1. La piomiositis es una enfermedad frecuente en nuestro medio.

2. Esta serie es hasta el momento no sólo la más grande publicada en Colombia sino en América.

3. En tres pacientes, el dolor muscular local sin tumefacción o masa acompañado de fiebre fue una forma más de presentación clínica.

4. Se identificó un caso de Pseudomonas aeruginosa como agente colonizador del músculo.

5. El antecedente traumático sólo se registró en un $18,9 \%$ de los casos.

6. La forma de presentarse clínicamente la enfermedad no siempre corresponde con la fase evolutiva descrita.

7. Independiente del germen causal, la enfermedad sigue un curso clínico semejante.

\section{Agradecimientos}

A la doctora Clara del Socorro Quintero Duque por la transcripción del manuscrito y al doctor Armando Rojas Cadena por su ayuda en la preparación de las tablas y figuras.

\section{Referencias}

1. Iglesias A, Ramírez LA. Piomiositis tropical. En: Bernal $\mathrm{J}$, ed. La infección osteoarticular. Armenia: Universidad del Quindío, Centro de Publicaciones, 1989:173. 
2. Miyake H. Beitrage zur kenntnis der sogennenta myositis infectiosa. Mitt a,d, Grezgeb Med Chir 1904;13:155.

3. Gellé G. Myosite suppureé suite de fatigue musculaire: infection purulente: mort. Bull Soc Anat Paris 1858; 33:414.

4. Scriba J. Beitrag zur aetiologie der myositis acuta. Deut Zeit Chir1885; 22:497.

5. Horn CV, Master S. Pyomyositis in Uganda. E Afr Med J 1968; 45:463.

6. De Salvia B. Myosite suppurada dos musculos rectos do abdomen:incisio:cura. Rev curso prat theor Fac Med Rio de Jan 1866; 3:49.

7. Kerrigan KR, Nelson SJ. Tropical pyomyositis in eastern Ecuador. Trans R Soc Trop Med Hyg 1992; 86:90.

8. Iglesias A, Pineda R. Piomiositis tropical. Acta Med Colomb 1980; 5:33.

9. Campbell S. Piomiositis. Informe de 75 pacientes. Biomédica 1991; Supl 1:75.

10. Rogers DW. Case of pyomyiositis ocurring in London. Br Med J 1973; 3:679.

11. Alavi MB, Smith EC, Latoo H. Pyomyositis tropical and non-tropical. N Engl J Med 1971; 294:854.

12. Chaitow J, Martur H, Knight P, Buchanan N. Pyomyositis tropicans: a diagnostic dilemma. Med J Austral 1980; 2:512.

13. Levin MJ,Gardner P,WaldongelFA. Tropical pyomyositis: an unusual infection due to Staphylococcus aureus. N Engl J Med 1971; 284:196.

14. Rodríguez A, Saravia J, Reyes A, Sierra F. Piomiositis tropical en el Hospital San Juan de Dios. Biomédica 1987; Sup 1:60.

15. Marcus RT, Foster WD. Observations on the clinical feature, aetiology and geographycal distribution of pyomyositis in east Africa. East Afr Med J 1968; 45:167.

16. Varela EA, Marulanda M, Hernández JI, González P. Piomiositis tropical. Acta Med Colomb 1988; 13:520.

17. Chiedozi L. Pyomyositis. Review of 205 cases in 112 patients. Am J Surg 1979; 137:255.

18. Ramírez LA, Ruiz E. Piomiositis en el Hospital San Juan de Dios, Armenia, Quindío. IATREIA 1990; 3:134.

19. Fanney D, Thomas LC, Schwartz E. An outbreak of pyomyositis in a large refugee camp in Thailand. Am J Trop Med Hyg 1982; 31:131.

20. Ashken MH, Cotton RE. Tropical skeletal muscle abscesses (pyomyositis tropicans). $\mathrm{Br} \mathrm{J}$ Surg 1963; 50:846.

21. Robin GC. Tropical myositis in Malaya. J Trop Med Hyg $1961 ; 64: 288$

22. Barón A, Rodríguez G. Piomiositis tropical. Estudio de tres pacientes. Acta Med Colomb 1980; 5:45.

23. Brown JD, Wheeler B. Pyomyositis. Report of 18 cases in Hawaii. Arch Intern Med 1984; 144:1749.

24. Sirinavin S, Mc Kracken G. Primary suppurative myositis in children. Am J Dis Children 1979; 133:263.
25. Eason R, Osbourne J, Ansford T, et al. Tropical pyomyositis in the Solomon Islands: clinical and aetiological feature. Trans R Soc Trop Med Hyg 1989; 83:275.

26. Alexander JW, Dionigi R, Meaking JL. Periodic variation in the antibacterial function of human neutrophils and its relationship to sepsis. Ann Surg 1971; 173.

27. Idoko JA, Oyeyinka GO, Giassudin AS, Naida A. Neutrophyl cell function and migration studies in Nigerian patients with tropical pyomyositis. J Infect 1987; 15:33.

28. Giasuddin AS, Idoko JA, Lamande RV. Serum immunoglobulin and complement levels in Nigerian patients with tropical pyomyositis (Letter). Trans R Soc Trop Med Hyg 1985; 79:564.

29. Giasuddin AS, Idoko JA, Lamande RV. Tropical pyomyositis: is it an immunodeficiency disease? Am J Trop Med Hyg 1986; 35:1231.

30. Hedstrom SA, Malinquist T. Sphingomyelinasa activity of Staphylococcus aureus strains from recurrent furunculosis and other infections. Acta Pathol Microbiol Inmunol Scand (B) 1982; 90:217.

31. Hedstrom SA, Nilsson-Ehle P. Trioleoylglycerollypolisis by Staphylococcus aureus strains from recurrent furunculosis, pyomyositis, impetigo and osteomyelitis. Acta Pathol Microbiol Inmunol Scand (B) 1983; 169:169.

32. Rollof J, Hedstrom SA, Nilsson-Ehle P. Lipolytic activity of Staphylococcus aureus strains from disseminated and localized infections. Acta Pathol Microbiol Inmunol Scand (B) 1987; 95:109.

33. Ramírez LA, Ruiz JE. Piomiositis. Acta Med Colomb 1988; 13(Supl):396.

34. Shepherd JJ. Miositis tropical: ¿Es una entidad? ¿Cuál es su causa? The Lancet (ed esp) 1984; 4:270.

35. Taylor JF, Templeton AC, Henderson B. Pyomyositis. A clinico-pathological study based on 19 autopsy cases, Mulago Hospital 1964-1968. E Afr Med J 1970; 47:494.

36. Adams E, Sigurdur G, Yocum D, et al. Streptococcal myositis. Arch Intern Med 1985; 145: 1020.

37. Schwab R, Panwalker AP. Klebsiella pyomyositis (Letter). Am J Med 1986; 81:1116.

38. Kratzke R, Golenbock D. Pyomyositis and hepatic abscess in association with Aeromonas hydrophila sepsis. Am J Med 1987; 347.

39. Swarts RL, Martínez LA, Robson HG. Gonococcal pyomyositis. JAMA 1981; 246:246.

40. Ramos A, Pérez J, Ruiz A y col. Piomiositis tropical complicada que semeja abdomen agudo. Rev Cub Med Trop 1985; 37:323.

41. Wendling $D$, Leaustic $M$, et al. Anaerobic cervical pyomyositis. Rev Rheun Mal Osteortric 1987; 54:59.

42. Brennessel DJ, Robbins N, Hindman S. Pyomyositis caused by Yersinia enterocolitica. J Clin Microbiol 1984; 20:293.

43. Sarubbi FA, Gafford GD, Bishop DR. Gram-negative bacterial pyomyositis: unique case and review. Rev Infect Dis 1989; 11:789. 
44. Gibson R, Rosenthal S, Lukert B. Pyomyositis increasing recognition in temperate climates. Am J Med 1984; 77:768.

45. Andy JJ, Ekpo EB. Cardiovascular complications of tropical pyomyositis. Trop Geogr Med 1987; 39:260.

46. Adeloye A. Intracranial suppuration complicating tropical pyomyositis. Report of two cases. Trans R Soc Trop Med Hyg 1982; 76:463.

47. Scrimgeour EM, Kaven J. Severe staphylococcal pneumonia complicating pyomyosistis. Am J Trop Med Hyg 1982; 31:822.

48. Taylor JF, Fluck D, Fluck D. Tropical myositis: ultrastructural studies. J Clin Path 1976; 29:1081.

49. Burkitt RT. Tropical pyomyositis. J Trop Med Hyg 1947; $5: 71$.

50. Jiménez-Mejía ME, Lozano de León F y col. Piomiositis por Staphylococcus aureus. Med Clin Bar 1992; 6: 201.

51. Schwartzman WA, et al. Staphylococcal pyomyositis in patients infected by the human immunodeficiency virus. Am J Med 1991; 5:595.
52. Halsted WS. Surgical papers. Baltimore: Johns Hopkins Press $1924 ; 103$.

53. DumaRJ, Weimberg AN, MedrekTF, et al. Streptococcal infections. A bacteriologic and clinical studies of streptococcal bacteremia. Medicine 1969; 48:87.

54. Churchil MA, Ceraci JE, Hunder GG. Musculosketal manifestations of bacterial endocarditis. Ann Intern Med $1977 ; 87: 754$.

55. Nishino H, Engl AG, Rima BK. Inclusion body myositis: the mumps virus hypothesis. Ann Neurol 1989; 25: 260.

56. Casademont J, Roger N, Pedrol E, et al. Streptococcal myositis as a complication of juvenile dermatomyositis. Neuromusc Dis 1991; 1:55.

57. Hall RL, Callaghan JJ, Moloney E, et al. Pyomyositis in a temperate climate. Presentation, diagnosis, and treatment. J Bone Joint Surg Am 1990; 72:1240.

58. Eggelmeijer F, Markusse HM, Stolk B, Bloem JL. Pyomyositis associated with Epstein-Barr virus infection [letter]. Br J Rheumatol 1988; 27:409. 